OPEN ACCESS

Edited by:

Richard Skipworth, University of Edinburgh,

United Kingdom

Reviewed by:

Markus Xie,

Genentech, United States

Yaqing Qie,

University of Texas MD Anderson

Cancer Center, United States

${ }^{*}$ Correspondence:

Xiaopeng $\mathrm{Hu}$

selihebe2019@163.com

Yu Zhang

wenruixue@hotmail.com

Specialty section:

This article was submitted to

Nutritional Immunology,

a section of the journal

Frontiers in Immunology

Received: 17 September 2021 Accepted: 09 November 2021 Published: 30 November 2021

Citation:

Xue W, Zhang $Y$, Wang $H$, Zhang $Y$ and HuX (2021) Multicenter Study of Controlling Nutritional Status (CONUT) Score as a Prognostic Factor in Patients With HIVRelated Renal Cell Carcinoma.

Front. Immunol. 12:778746. doi: 10.3389/fimmu.2021.778746

\section{Multicenter Study of Controlling Nutritional Status (CONUT) Score as a Prognostic Factor in Patients With HIV-Related Renal Cell Carcinoma}

\author{
Wenrui Xue ${ }^{1}$, Yu Zhang ${ }^{2}$, Hua Wang $^{3}$, Yu Zhang ${ }^{1 *}$ and Xiaopeng $\mathrm{Hu}^{4 *}$ \\ ${ }^{1}$ Beijing Youan Hospital, Capital Medical University, Beijing, China, ${ }^{2}$ Beijing Ditan Hospital, Capital Medical University, Beijing, \\ China, ${ }^{3}$ Chengdu Public Health Clinical Medical Center, Sichuan, China, ${ }^{4}$ Beijing Chaoyang Hospital, Capital Medical \\ University, Beijing, China
}

Objective: In recent years, the controlled nutritional status (CONUT) score has been widely recognized as a new indicator for assessing survival in patients with urological neoplasms, including renal, ureteral, and bladder cancer. However, the CONUT score has not been analyzed in patients with HIV-related urological neoplasms. Therefore, we aimed to evaluate the prognostic significance of the CONUT score in patients with HIV-related renal cell carcinoma (RCC).

Methods: A total of 106 patients with HIV-related RCC were recruited from four hospitals between 2012 and 2021, and all included patients received radical nephrectomy or partial nephrectomy. The CONUT score was calculated by serum albumin, total lymphocyte counts, and total cholesterol concentrations. Patients with RCC were divided into two groups according to the optimal cutoff value of the CONUT score. Survival analysis of different CONUT groups was performed by the Kaplan-Meier method and a log rank test. A Cox proportional risk model was used to test for correlations between clinical variables and cancer-specific survival (CSS), overall survival (OS), and disease-free survival (DFS). Clinical variables included age, sex, hypertension, diabetes, tumor grade, Fuhrman grade, histology, surgery, and CD4+ T lymphocyte count.

Result: The median age was 51 years, with 93 males and 13 females. At a median followup of 41 months, 25 patients (23.6\%) had died or had tumor recurrence and metastasis. The optimal cutoff value for the CONUT score was 3, and a lower CONUT score was associated with the Fuhrman grade $(P=0.024)$. Patients with lower CONUT scores had better CSS (HR 0.197, 95\% Cl 0.077-0.502, P=0.001), OS (HR 0.177, 95\% Cl 0.0700.446, $\mathrm{P}<0.001)$ and DFS (HR 0.176, 95\% Cl 0.070-0.444, $\mathrm{P}<0.001)$. Multivariate Cox regression analysis indicated that a low CONUT score was an independent predictor of CSS, OS and DFS (CSS: HR=0.225, 95\% Cl 0.067-0.749, P=0.015; OS: HR=0.201, 95\% Cl 0.061-0.661, $\mathrm{P}=0.008$; DFS: HR=0.227, 95\% Cl 0.078-0.664, $\mathrm{P}=0.007)$. In addition, a low Fuhrman grade was an independent predictor of CSS (HR 0.192, 95\% Cl 0.0450.810, $\mathrm{P}=0.025)$, OS (HR 0.203, 95\% Cl 0.049-0.842, $\mathrm{P}=0.028$ ), and DFS (HR 0.180, $95 \% \mathrm{Cl}$ 0.048-0.669, $\mathrm{P}=0.010$ ), while other factors, such as age, sex, hypertension, 
diabetes, tumor grade, histology, surgery, and CD4+ T lymphocyte count, were not associated with survival outcome.

Conclusion: The CONUT score, an easily measurable immune-nutritional biomarker, may provide useful prognostic information in HIV-related RCC.

Keywords: controlling nutritional status score, HIV-related renal cell carcinoma, prognostic factor, cancer-specific survival (CSS), overall survival (OS), disease-free survival (DFS), highly active antiretroviral therapy (HAART)

\section{INTRODUCTION}

RCC is the most common pathologic type of renal cancer and the seventh most common tumor, accounting for $2 \%$ to $3 \%$ of all cancers (1). Twenty percent of newly diagnosed RCC patients have advanced disease, and approximately $30 \%$ experience local or distant disease recurrence after surgery for localized RCC (2). In recent years, relatively few infection cases of HIV-related RCC have been reported worldwide. Patients with such RCC have concurrent immune infection. Human immunodeficiency virus (HIV) infects human dendritic cells and macrophages and activates CD4+ $\mathrm{T}$ lymphocytes, resulting in disruption of the immune system, so the tumor incidence and mortality differ from those in ordinary RCC patients (3). RCC is more common in HIV-infected individuals than in age-matched non-HIVinfected individuals and is a common cause of morbidity and mortality. Possible mechanisms for this increased risk include reduced immune surveillance, direct effects of viral proteins, or cytokine dysregulation $(4,5)$. With the widespread application of and tremendous progress in early activation of highly active antiretroviral therapy (HAART), both virological suppression and immune recovery in patients with HIV-related RCC have been maintained at a good level (6). However, non-AIDSdefining cancers (non-ADCs), including urinary cancers, anal cancers, lung cancers, breast cancers and skin cancers, are still three times more frequent (7). If patients with HIV-related RCC can be assessed early, their survival could be significantly improved. Therefore, it is important to develop off-the-shelf biomarkers that can predict and even modify tumor outcomes based on risk stratification (8).

Immunological status comprising inflammatory and nutritional status, remains an important predictor of prognosis in patients with malignant tumors (9). Several biomarkers, such as the prognostic nutritional index (PNI) and the neutrophil to lymphocyte ratio (NLR), have been reported to be independent prognostic factors (10-12). Recently, the CONUT score, which is calculated from serum albumin, total lymphocyte counts, and total cholesterol concentration, has gained attention as a biomarker for predicting survival in patients with multiple cancers. A high CONUT score means lower levels of albumin, lymphocytes, and cholesterol, which are often associated with poorer nutritional and immune status in patients and may lead to poorer survival (13). Maintaining optimal nutritional status can greatly improve quality of life while reducing comorbidities, progression of HIV infection, and HIV-related mortality (14, 15). In addition, good nutrition also helps HIV-infected patients absorb HIV drugs (16). The effects of poor nutritional status and
HIV are synergistic and interrelated, thus amplifying their respective harmful effects $(15,17)$. Increasing evidence suggests that, in addition to the genetic basis, host nutritional status and inflammatory responses also play an important role in cancer development and progression (18). At present, some articles suggest that the CSS, OS and DFS of patients with 5-year ordinary RCC (non-HIV related) in the low-CONUT group are significantly higher than those in the high-CONUT group (8, 19-24), but some articles suggest that a high CONUT score is not related to the prognosis of patients with ordinary RCC (25). However, there are no articles about the relationship between CONUT score and HIV-related RCC. To the best of our knowledge, this is the first multicenter study to evaluate the prognostic value of the CONUT score in HIV-related RCC.

\section{MATERIALS AND METHODS}

\section{Patients}

We performed an open-label, retrospective, multicenter, cohort study. A total of 106 patients with HIV-related RCC who underwent radical nephrectomy or partial nephrectomy were included. All participants underwent preoperative urological CT examination showing a renal carcinoma volume $\leq 7 \mathrm{~cm}$ between 2012 and 2021. We excluded patients with ordinary RCC without HIV infection, patients with lymph node metastasis or distant metastasis, and patients with no follow-up results. All enrolled patients had provided blood samples with results for serum albumin, total lymphocyte counts, and total cholesterol concentration one week before surgery and were treated with HAART and monitored for associated CD4+ T lymphocyte count.

Pathological stage was determined according to the 2010 TNM grade and tumor grade according to the Fuhrman grading system. This study was in accordance with the Helsinki Declaration and approved by the Ethics Review Committee of all included hospitals. During follow-up, patients or their next of kin were informed of the study in detail, and verbal consent was obtained. All data are kept confidential.

\section{Follow-Up}

Every three months within the first 3 years after surgery, the patient was admitted to the outpatient department of the hospital for routine blood examination, blood biochemistry, chest X-ray, abdominal color Doppler ultrasound and enhanced urinary CT examination. After 3 years, the above review was performed every 6 months until tumor recurrence, metastasis or death. Relapse is equal to the first detection of local recurrence, 
and metastasis is equal to the first discovery of lymph node or distant organ metastases (lung metastasis, brain metastasis, liver metastasis, etc.). Death was confirmed by relevant information from the hospitals or notification by the patient's family during telephone follow-up.

\section{Study Endpoints}

We considered CSS, OS, and DFS as the end points of the study (in months). CSS was defined as the time from the date of surgery to cancer-related death. OS was defined as the time from the date of surgery to the death of the individual from any cause. DFS was defined as the time from the date of surgery to radiologically or histologically confirmed recurrence or metastasis.

\section{CONUT Score, PNI and NLR}

The CONUT score was calculated by serum albumin, total lymphocyte counts, and total cholesterol concentration (Table 1). The optimal cutoff value of the CONUT score was determined using the receiver operating characteristic (ROC) curve and the maximum Youden index value. The PNI was calculated as $10 \times$ serum albumin $(\mathrm{g} / \mathrm{dl})+0.005 \times$ total lymphocyte count (per $\mathrm{mm} 3$ ). The NLR was calculated as the ratio of the number of neutrophils to the number of lymphocytes.

\section{Statistics}

A chi-square test was used to analyze the correlations between the CONUT score and variables including age, sex, hypertension, diabetes, tumor grade, Fuhrman grade, histology, surgery, and CD4+ T lymphocyte count. Kaplan-Meier survival curves were plotted to estimate CSS, OS, and DFS. The predictors of CSS, OS and DFS were determined by univariate analysis, a Cox proportional risk model was used for multivariate analysis evaluation, and variables with $\mathrm{P}<0.05$ in univariate analysis were included in subsequent multivariate analysis. GraphPad Prism Version 9 (GraphPad Software, La Jolla California USA, www.graphpad.com) was used to generate survival curves. Statistical analysis and ROC curves mapping were performed using SPSS version 23 (SPSS Inc., Chicago, IL, USA).

\section{RESULTS}

\section{CONUT Score and Its Cutoff Value}

According to ROC analysis, the Youden index was used to determine the optimal cutoff value of the CONUT score as 3
(AUC: $0.746,95 \%$ CI: $0.638-0.855, \mathrm{P}<0.001$, Figure 1. The area under the receiver operating characteristics curve, AUC). The CONUT score was assessed by dichotomous variables (low: $<3$, high: $\geq 3$ ).

\section{Clinicopathological Features}

Of the 106 patients enrolled, with a median age of 51 (IQR 27-75) years at the time of surgery, 93 were males, 13 were females, 80 underwent radical nephrectomy, and 26 underwent partial nephrectomy. Among them, 104 cases were clear cell carcinoma, 87 cases were T1N0M0, and 19 cases were T3N0M0. For the Fuhrman classification, 65 were grades I-II, and 41 were grades IIIIV. The median CD4+ T lymphocyte count value was 435 (IQR 48-1536) cells/ $\mu \mathrm{l}$. The highest preoperative viral load was 1,018,049 copies/mL, and the lowest was undetectable (Table 2). The CONUT score was high in 45 cases (42\%) and low in 61 cases (58\%), and was closely correlated with the Fuhrman grade. A low CONUT score was significantly associated with lower Fuhrman grade (I-II vs III-IV, $66.2 \%$ vs $43.9 \%$, respectively, $\mathrm{p}=0.024$ ). The CONUT score had no significant correlation with age, sex, hypertension, diabetes, tumor grade, histology, surgery, or CD4+ T lymphocyte count $(\mathrm{P}>0.05)$ (Table 3 ).

\section{Survival Outcome}

The median postoperative follow-up time of CSS and OS was 41 (IQR 6-105) months, and that of DFS was 41 (IQR 4-105) months. In the high- and low-CONUT groups, the 5-year CSS rates were $47.79 \%$ and $85.54 \%(\mathrm{P}<0.001)$ (Figure $2 \mathrm{~A})$, the 5 -year OS rates were $44.47 \%$ and $85.54 \%(\mathrm{P}<0.001)$ (Figure 2B), and the 5-year DFS rates were $44.86 \%$ and $86.16 \%(\mathrm{P}<0.001)$ (Figure 2C), respectively.

As shown in Table 4, patients with lower CONUT scores had better CSS (HR 0.197, 95\% CI 0.077-0.502, P=0.001), OS (HR $0.177,95 \%$ CI $0.070-0.446, \mathrm{P}<0.001$ ) and DFS (HR 0.176, 95\% CI $0.070-0.444, \mathrm{P}<0.001)$. In addition, Fuhrman grade was also significantly correlated with CSS, OS and DFS $(\mathrm{P}<0.01)$. Multivariate Cox regression analysis indicated that a low CONUT score was an independent predictor of CSS, OS and DFS (CSS: $\mathrm{HR}=0.225$, 95\% CI 0.067-0.749, $\mathrm{P}=0.015$; OS: $\mathrm{HR}=0.201$, 95\% CI 0.061-0.661, $\mathrm{P}=0.008$; DFS: $\mathrm{HR}=0.227,95 \%$ CI $0.078-0.664, \mathrm{P}=0.007)$. A low Fuhrman grade was an independent predictor of CSS (HR 0.192, 95\% CI 0.045-0.810, $\mathrm{P}=0.025)$, OS (HR 0.203, 95\% CI 0.049-0.842, $\mathrm{P}=0.028$ ), and DFS (HR 0.180, 95\% CI 0.048-0.669, $\mathrm{P}=0.010$ ) (Table 4), while other factors, such as age, sex, hypertension, diabetes, tumor grade,

TABLE 1 | Definition of CONUT score.

\begin{tabular}{|c|c|c|c|c|}
\hline \multirow[t]{2}{*}{ Parameters } & \multicolumn{4}{|c|}{ CONUT } \\
\hline & Normal & Light & Moderate & Severe \\
\hline Serum albumin (g/dL) & $\geq 3.50$ & $3.00-3.49$ & 2.50-2.99 & $<2.50$ \\
\hline Score & 0 & 2 & 4 & 6 \\
\hline Total lymphocyte $\left(/ \mathrm{mm}^{3}\right)$ & $\geq 1600$ & 1200-1599 & 800-1199 & $<800$ \\
\hline Score & 0 & 1 & 2 & 3 \\
\hline Total cholesterol (mg/dL) & $\geq 180$ & $140-179$ & $100-139$ & $<100$ \\
\hline Score & 0 & 1 & 2 & 3 \\
\hline CONUT score (total) & $0-1$ & $2-4$ & $5-8$ & $9-12$ \\
\hline
\end{tabular}




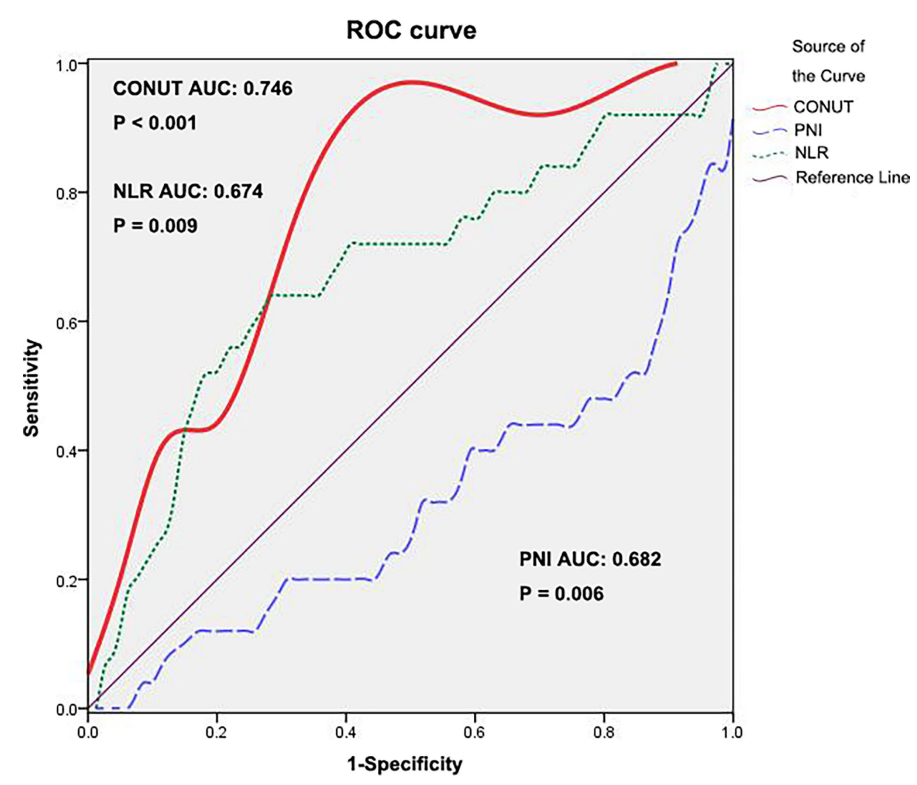

FIGURE 1 | ROC curve for CONUT, PNI and NLR. ROC, receiver operating characteristic; CONUT, controlling nutritional status; PNI, prognostic nutritional index; NLR, neutrophil-lymphocyte ratio.

histology, surgery, and CD4+ T lymphocyte count, were not associated with survival outcome.

\section{Compare CONUT Score With Other Biomarkers in Patients With HIV-Related RCC for Survival Prediction}

The PNI and NLR values of 106 patients are also shown in Table 2. The ROC curve with the most sensitive and specific cutoff values of PNI and NLR is also shown in Figure 1. We compared the AUCs for predicting 5-year OS by CONUT, PNI and NLR. Among the prognostic factors, the CONUT has the highest AUC (0.746). The AUC score of PNI and NLR in relation to 5-year OS was 0.682 (95\% CI 0.553-0.811) and 0.674 (95\% CI $0.545-0.803)$, respectively.

\section{DISCUSSION}

In this study, patients with surgically treated HIV-related RCC with high CONUT scores had significantly shorter CSS, OS, and DFS than patients with low CONUT scores. Multivariate analysis further showed that the CONUT score was an independent factor influencing these survival outcomes. In addition, a low Fuhrman grade was significantly associated with survival outcomes. This study is the first to study the prognostic factors of patients with HIV-related RCC, which has certain reference significance for the surgical treatment selection of patients with HIV infection and the prognosis of patients with HIV-related RCC.

Among people living with HIV, non-HIV-related morbidity and mortality are becoming more common, and non-HIV hypotoxicity is becoming an important source of mortality (26).
In recent years, there has been a significant increase in the incidence of malignant tumors in HIV-infected people because of the increased life expectancy associated with HAART, and urologists are increasingly likely to encounter HIV-infected patients with the same urinary problems as the general population (4). Strictly evaluating the surgical indications of patients with HIV-related RCC and early surgical treatment are crucial for patient prognosis. HIV mainly invades human CD4+ T lymphocytes, causing a reduction in their number and functional defects, thereby resulting in low immune function and an increasing incidence of various opportunistic infections. Although early literature on surgical outcomes in HIV-positive patients suggested an increased risk of perioperative complications (27), recent studies have shown that most procedures can be performed safely in HIV-positive patients with appropriate preoperative evaluation of CD4+ T lymphocyte count and viral load (28). Therefore, preoperative routine examination of CD4+ T lymphocytes in patients with HIV-related RCC is an aspect of evaluating whether patients can tolerate surgery, but CD4+ T lymphocytes do not serve as a good predictor of the survival prognosis of these patients; therefore, it is necessary to find prognostic predictors for patients with HIV-related RCC.

The CONUT score was determined by serum albumin, total lymphocyte counts, and total cholesterol concentration. Serum albumin is an indicator that can reflect patients' nutritional status and is closely related to patients' surgical tolerance and postoperative recovery. A lower level of serum albumin means the loss of immunity (29). HIV RNA levels and CD4+ T lymphocyte counts provide some prognostic information about HIV disease progression, but data published by Shruti $\mathrm{H}$ Mehta suggest that serum albumin levels provide more prognostic 
TABLE 2 | Basic information of the cases and HIV- related data.

\begin{tabular}{|c|c|c|c|c|c|c|c|c|c|c|c|c|}
\hline Case & Age & Gender & $\begin{array}{l}\text { Fuhr- } \\
\text { man }\end{array}$ & Tumor grade & Histology & Comorbidity & $\begin{array}{l}\text { CD4 count } \\
\text { (cells/ul) }\end{array}$ & $\begin{array}{c}\text { Viral load } \\
\text { (copies/ml) }\end{array}$ & Surgery & CONUT & PNI & NLR \\
\hline 1 & 59 & Male & III-IV & T1NOMO & Clear cell & Hypertension & 614 & NT & $\mathrm{RN}$ & 1 & 54.75 & 0.9628 \\
\hline 2 & 74 & Male & $|-| \mid$ & T1N0MO & Clear cell & Hypertension & 356 & NT & $\mathrm{RN}$ & 2 & 41.65 & 2.7287 \\
\hline 3 & 43 & Male & $|-| \mid$ & T1N0M0 & Clear cell & None & 400 & TND & $\mathrm{RN}$ & 2 & 47.65 & 1.6163 \\
\hline 4 & 58 & Male & $|-| \mid$ & T1N0M0 & Clear cell & None & 1536 & 341 & $\mathrm{RN}$ & 5 & 45.75 & 2.3498 \\
\hline 5 & 54 & Male & III-IV & T1NOMO & Clear cell & Hypertension & 628 & NT & $\mathrm{RN}$ & 1 & 52.75 & 1.7355 \\
\hline 6 & 51 & Male & $|-| \mid$ & T1NOMO & Clear cell & Diabetes & 291 & TND & $\mathrm{RN}$ & 1 & 52.3 & 2.5505 \\
\hline 7 & 54 & Male & $|-| \mid$ & T1NOMO & Clear cell & $\begin{array}{l}\text { Hypertension+ } \\
\text { Diabetes }\end{array}$ & 880 & TND & $P N$ & 0 & 58.05 & 1.0433 \\
\hline 8 & 51 & Male & III-IV & T3NOMO & Clear cell & None & 190 & 6495 & $\mathrm{RN}$ & 2 & 49.4 & 1.9253 \\
\hline 9 & 34 & Male & III-IV & T3NOMO & Clear cell & None & 462 & TND & $\mathrm{RN}$ & 3 & 49.55 & 2.9802 \\
\hline 10 & 72 & Male & III-IV & T1N0M0 & Clear cell & None & 378 & NT & $\mathrm{RN}$ & 2 & 47.65 & 3.6067 \\
\hline 11 & 54 & Female & III-IV & T1N0M0 & Clear cell & None & 535 & 63188 & $\mathrm{RN}$ & 1 & 48.65 & 1.4571 \\
\hline 12 & 51 & Male & $|-| \mid$ & T1NOMO & Clear cell & None & 142 & 409601 & $\mathrm{RN}$ & 1 & 47.25 & 0.8667 \\
\hline 13 & 53 & Male & $|-| \mid$ & T1NOMO & $\begin{array}{l}\text { Non-clear } \\
\text { cell }\end{array}$ & Hypertension & 328 & 31164 & $\mathrm{RN}$ & 0 & 52.85 & 2.0675 \\
\hline 14 & 55 & Male & III-IV & T1N0M0 & Clear cell & None & 420 & $<40$ & $\mathrm{RN}$ & 1 & 49.1 & 2.8654 \\
\hline 15 & 39 & Male & $|-| \mid$ & T1NOMO & Clear cell & Hypertension & 190 & TND & $\mathrm{RN}$ & 0 & 47.65 & 1.5698 \\
\hline 16 & 46 & Male & $|-| \mid$ & T1N0M0 & Clear cell & Hypertension & 267 & 61 & $\mathrm{RN}$ & 0 & 52.5 & 3.0571 \\
\hline 17 & 67 & Male & III-IV & T1N0M0 & Clear cell & Diabetes & 821 & TND & $\mathrm{RN}$ & 2 & 43.6 & 4.2982 \\
\hline 18 & 59 & Male & III-IV & T1NOMO & Clear cell & None & 375 & TND & $\mathrm{RN}$ & 3 & 52.25 & 2.8956 \\
\hline 19 & 56 & Male & $|-| \mid$ & T3NOMO & Clear cell & None & 495 & TND & PN & 2 & 51.5 & 3.0782 \\
\hline 20 & 54 & Female & $|-| \mid$ & T1NOMO & Clear cell & None & 687 & 552 & $\mathrm{RN}$ & 0 & 55.25 & 1.9875 \\
\hline 21 & 62 & Male & III-IV & T1NOMO & Clear cell & Hypertension & 325 & NT & $\mathrm{RN}$ & 3 & 40.5 & 3.8593 \\
\hline 22 & 49 & Male & III-IV & T1NOMO & Clear cell & None & 641 & $<40$ & $\mathrm{RN}$ & 1 & 51.25 & 0.9889 \\
\hline 23 & 66 & Male & $|-| \mid$ & T1NOMO & Clear cell & Diabetes & 531 & 180 & $\mathrm{RN}$ & 2 & 44.25 & 2.9136 \\
\hline 24 & 30 & Female & $|-| \mid$ & T1NOMO & Clear cell & None & 604 & TND & PN & 2 & 51.25 & 1.0404 \\
\hline 25 & 39 & Male & $|-| \mid$ & T1N0M0 & Clear cell & None & 296 & NT & $\mathrm{RN}$ & 1 & 53.8 & 0.9825 \\
\hline 26 & 51 & Male & III-IV & T1NOMO & Clear cell & None & 151 & 73907 & $\mathrm{RN}$ & 3 & 45.85 & 1.2980 \\
\hline 27 & 50 & Male & $|-| \mid$ & T1NOMO & Clear cell & None & 327 & 4909 & $\mathrm{RN}$ & 2 & 48.95 & 2.7248 \\
\hline 28 & 50 & Male & III-IV & T1NOMO & Clear cell & None & 665 & TND & $\mathrm{RN}$ & 2 & 55.1 & 1.1713 \\
\hline 29 & 48 & Male & III-IV & T1NOMO & Clear cell & None & 589 & TND & $\mathrm{PN}$ & 2 & 52.15 & 1.2731 \\
\hline 30 & 57 & Male & III-IV & T1NOMO & Clear cell & Diabetes & 1062 & $<40$ & $\mathrm{RN}$ & 0 & 60.9 & 0.8381 \\
\hline 31 & 52 & Male & $|-| \mid$ & T1NOMO & Clear cell & Hypertension & 268 & TND & $\mathrm{RN}$ & 0 & 56.9 & 1.1234 \\
\hline 32 & 36 & Male & $|-| \mid$ & T1NOMO & Clear cell & Diabetes & 140 & 174 & $\mathrm{RN}$ & 3 & 46.75 & 3.7321 \\
\hline 33 & 32 & Male & $|-| \mid$ & T1N0M0 & Clear cell & None & 1229 & TND & $\mathrm{RN}$ & 3 & 47.95 & 6.4360 \\
\hline 34 & 51 & Male & III-IV & T3NOMO & Clear cell & None & 589 & TND & $\mathrm{RN}$ & 2 & 54.9 & 3.0312 \\
\hline 35 & 65 & Male & III-IV & T1NOMO & Clear cell & None & 237 & 2241 & $\mathrm{RN}$ & 5 & 41.3 & 3.7697 \\
\hline 36 & 50 & Male & III-IV & T3NOMO & Clear cell & None & 179 & 57922 & $\mathrm{RN}$ & 2 & 41.4 & 3.3089 \\
\hline 37 & 27 & Male & $|-| \mid$ & T1NOMO & Clear cell & None & 1082 & TND & PN & 2 & 44.3 & 4.0905 \\
\hline 38 & 43 & Female & $|-| \mid$ & T1NOMO & Clear cell & None & 538 & TND & PN & 6 & 37 & 5.0417 \\
\hline 39 & 46 & Male & $|-| \mid$ & T3NOMO & Clear cell & None & 567 & $<40$ & $\mathrm{RN}$ & 2 & 45.65 & 1.1357 \\
\hline 40 & 63 & Male & III-IV & T3NOMO & Clear cell & None & 73 & 1018049 & $\mathrm{RN}$ & 8 & 32.6 & 5.0417 \\
\hline 41 & 51 & Male & $|-| \mid$ & T1N0MO & Clear cell & None & 537 & 77565 & $\mathrm{RN}$ & 1 & 54.6 & 1.2692 \\
\hline 42 & 35 & Male & $|-| \mid$ & T1NOMO & Clear cell & None & 223 & TND & PN & 2 & 46.15 & 1.3043 \\
\hline 43 & 52 & Male & $|-| \mid$ & T1NOMO & Clear cell & None & 321 & 14317 & PN & 3 & 52.3 & 1.6026 \\
\hline 44 & 61 & Male & III-IV & T3NOMO & Clear cell & None & 148 & 34900 & $\mathrm{RN}$ & 7 & 36.28 & 2.5641 \\
\hline 45 & 41 & Male & $|-| \mid$ & T1NOMO & Clear cell & None & 48 & 59900 & $\mathrm{RN}$ & 4 & 44.55 & 1.5039 \\
\hline 46 & 55 & Male & $|-| \mid$ & T1NOMO & Clear cell & Diabetes & 438 & TND & PN & 4 & 43.25 & 2.4747 \\
\hline 47 & 47 & Male & $|-| \mid$ & T1N0M0 & Clear cell & None & 530 & TND & PN & 2 & 45.1 & 1.2083 \\
\hline 48 & 54 & Female & $|-| \mid$ & T1NOMO & Clear cell & None & 234 & TND & $\mathrm{RN}$ & 6 & 38.1 & 2.6764 \\
\hline 49 & 61 & Female & $|-| \mid$ & T1N0M0 & Clear cell & None & 311 & TND & $\mathrm{RN}$ & 4 & 40.85 & 3.0689 \\
\hline 50 & 75 & Male & $|-| \mid$ & T1N0M0 & Clear cell & Hypertension & 180 & TND & $\mathrm{PN}$ & 5 & 45.55 & 3.0093 \\
\hline 51 & 67 & Male & $|-| \mid$ & T1NOMO & $\begin{array}{l}\text { Non-clear } \\
\text { cell }\end{array}$ & None & 356 & TND & $\mathrm{RN}$ & 4 & 40.25 & 3.3926 \\
\hline 52 & 57 & Male & III-IV & T1NOMO & Clear cell & $\begin{array}{l}\text { Hypertension+ } \\
\text { Diabetes }\end{array}$ & 1399 & TND & $\mathrm{RN}$ & 1 & 49.05 & 1.4150 \\
\hline 53 & 45 & Male & III-IV & T3NOMO & Clear cell & None & 530 & TND & $\mathrm{RN}$ & 6 & 38.7 & 5.0176 \\
\hline 54 & 39 & Male & $|-| \mid$ & T1NOMO & Clear cell & None & 335 & TND & $\mathrm{PN}$ & 3 & 39.3 & 4.6872 \\
\hline 55 & 63 & Male & $|-| \mid$ & T1NOMO & Clear cell & None & 423 & 4528 & $\mathrm{RN}$ & 2 & 46.5 & 2.9630 \\
\hline 56 & 41 & Male & III-IV & T1NOMO & Clear cell & None & 741 & TND & $\mathrm{RN}$ & 5 & 37.27 & 4.9724 \\
\hline 57 & 48 & Male & $|-| \mid$ & T1NOMO & Clear cell & None & 552 & $<40$ & $\mathrm{RN}$ & 1 & 47.5 & 2.5657 \\
\hline 58 & 68 & Male & III-IV & T1NOMO & Clear cell & None & 269 & TND & PN & 2 & 50.9 & 3.0171 \\
\hline
\end{tabular}

(Continued) 
TABLE 2 | Continued

\begin{tabular}{|c|c|c|c|c|c|c|c|c|c|c|c|c|}
\hline Case & Age & Gender & $\begin{array}{l}\text { Fuhr- } \\
\text { man }\end{array}$ & Tumor grade & Histology & Comorbidity & $\begin{array}{l}\text { CD4 count } \\
\text { (cells/ul) }\end{array}$ & $\begin{array}{l}\text { Viral load } \\
\text { (copies/ml) }\end{array}$ & Surgery & CONUT & PNI & NLR \\
\hline 59 & 46 & Female & III-IV & T3NOMO & Clear cell & None & 412 & TND & $\mathrm{RN}$ & 2 & 50.25 & 1.5623 \\
\hline 60 & 36 & Male & III-IV & T1NOMO & Clear cell & None & 637 & 380 & $\mathrm{RN}$ & 6 & 32.28 & 3.9743 \\
\hline 61 & 55 & Male & $|-| \mid$ & T3NOMO & Clear cell & None & 190 & TND & $\mathrm{RN}$ & 2 & 43.9 & 3.0587 \\
\hline 62 & 38 & Male & III-IV & T1N0MO & Clear cell & None & 423 & TND & $\mathrm{RN}$ & 3 & 46.9 & 3.2568 \\
\hline 63 & 64 & Male & III-IV & T1NOMO & Clear cell & None & 449 & 900 & $\mathrm{RN}$ & 5 & 41.7 & 3.4102 \\
\hline 64 & 69 & Male & $|-| \mid$ & T1NOMO & Clear cell & None & 432 & TND & $\mathrm{RN}$ & 1 & 52.15 & 3.8576 \\
\hline 65 & 51 & Male & III-IV & T3NOMO & Clear cell & None & 547 & 82080 & $\mathrm{RN}$ & 2 & 45.2 & 1.4568 \\
\hline 66 & 49 & Female & $|-| \mid$ & T1N0M0 & Clear cell & None & 734 & TND & $\mathrm{RN}$ & 3 & 41.15 & 3.7596 \\
\hline 67 & 38 & Male & $|-| \mid$ & T1NOMO & Clear cell & None & 353 & TND & PN & 2 & 43.9 & 4.3264 \\
\hline 68 & 55 & Male & $|-| \mid$ & T1NOMO & Clear cell & None & 229 & 24611 & PN & 2 & 50.4 & 2.6384 \\
\hline 69 & 46 & Male & $|-| \mid$ & T1NOMO & Clear cell & None & 431 & TND & PN & 3 & 39.9 & 4.7919 \\
\hline 70 & 61 & Female & $|-| \mid$ & T1NOMO & Clear cell & None & 524 & TND & $\mathrm{RN}$ & 5 & 39.4 & 4.5244 \\
\hline 71 & 61 & Male & III-IV & T1NOMO & Clear cell & None & 421 & $<40$ & $\mathrm{RN}$ & 5 & 38.43 & 3.1028 \\
\hline 72 & 49 & Male & $|-| \mid$ & T3NOMO & Clear cell & None & 778 & $<40$ & PN & 3 & 49.8 & 2.0970 \\
\hline 73 & 33 & Male & $|-| \mid$ & T1NOM0 & Clear cell & Diabetes & 567 & TND & PN & 4 & 36.8 & 4.5654 \\
\hline 74 & 64 & Male & $|-| \mid$ & T3NOMO & Clear cell & None & 258 & 3600 & $\mathrm{RN}$ & 6 & 38.75 & 4.1971 \\
\hline 75 & 57 & Male & $|-| \mid$ & T1NOMO & Clear cell & None & 655 & TND & $\mathrm{RN}$ & 4 & 46.05 & 3.2347 \\
\hline 76 & 54 & Male & $|-| \mid$ & T1NOMO & Clear cell & None & 356 & TND & $\mathrm{RN}$ & 1 & 48.1 & 1.6593 \\
\hline 77 & 42 & Male & $|-| \mid$ & T1NOMO & Clear cell & None & 551 & $<40$ & $\mathrm{RN}$ & 2 & 45.6 & 2.6567 \\
\hline 78 & 45 & Male & $|-| \mid$ & T1NOMO & Clear cell & None & 332 & TND & $\mathrm{RN}$ & 1 & 46.65 & 3.0105 \\
\hline 79 & 53 & Male & III-IV & T3NOMO & Clear cell & None & 533 & TND & $\mathrm{RN}$ & 6 & 40.6 & 3.5971 \\
\hline 80 & 66 & Male & $|-| \mid$ & T1NOMO & Clear cell & None & 550 & TND & PN & 3 & 42.9 & 3.1775 \\
\hline 81 & 41 & Female & $|-| \mid$ & T1NOMO & Clear cell & Diabetes & 477 & TND & $\mathrm{RN}$ & 2 & 45.4 & 2.8861 \\
\hline 82 & 45 & Male & $|-| \mid$ & T1NOMO & Clear cell & None & 377 & TND & $\mathrm{RN}$ & 3 & 48.05 & 1.2693 \\
\hline 83 & 55 & Male & III-IV & T1NOMO & Clear cell & None & 636 & 5352 & $\mathrm{RN}$ & 2 & 43.75 & 2.2327 \\
\hline 84 & 70 & Male & III-IV & T3NOMO & Clear cell & Hypertension & 359 & TND & $\mathrm{RN}$ & 3 & 48.1 & 2.4647 \\
\hline 85 & 48 & Male & III-IV & T3NOMO & Clear cell & None & 678 & TND & $\mathrm{RN}$ & 2 & 45.15 & 3.6537 \\
\hline 86 & 50 & Male & $|-| \mid$ & T1NOMO & Clear cell & None & 790 & TND & $\mathrm{RN}$ & 1 & 48.2 & 2.6974 \\
\hline 87 & 44 & Male & $|-| \mid$ & T1NOMO & Clear cell & None & 358 & TND & $\mathrm{RN}$ & 2 & 48 & 1.2387 \\
\hline 88 & 29 & Male & $|-| \mid$ & T1NOMO & Clear cell & None & 559 & NT & PN & 2 & 48.3 & 3.4697 \\
\hline 89 & 68 & Male & $|-| \mid$ & T1NOMO & Clear cell & None & 243 & TND & $\mathrm{RN}$ & 5 & 39.15 & 4.6874 \\
\hline 90 & 49 & Female & $|-| \mid$ & T1NOMO & Clear cell & None & 438 & TND & PN & 1 & 44.55 & 2.5813 \\
\hline 91 & 38 & Male & III-IV & T1N0M0 & Clear cell & None & 457 & $<40$ & $\mathrm{RN}$ & 7 & 36.6 & 4.6687 \\
\hline 92 & 51 & Male & $|-| \mid$ & T1NOMO & Clear cell & None & 221 & TND & $\mathrm{RN}$ & 4 & 39.45 & 4.4367 \\
\hline 93 & 33 & Male & $|-| \mid$ & T1N0M0 & Clear cell & Hypertension & 573 & 56781 & $\mathrm{RN}$ & 3 & 45.95 & 2.1187 \\
\hline 94 & 67 & Male & III-IV & T1NOMO & Clear cell & None & 329 & TND & $\mathrm{RN}$ & 3 & 39.45 & 4.7652 \\
\hline 95 & 56 & Male & $|-| \mid$ & T1N0M0 & Clear cell & None & 343 & 2538 & $\mathrm{RN}$ & 2 & 47.25 & 1.1495 \\
\hline 96 & 48 & Male & $|-| \mid$ & T1NOMO & Clear cell & None & 431 & TND & PN & 1 & 49.45 & 2.7465 \\
\hline 97 & 31 & Male & III-IV & T1N0M0 & Clear cell & None & 415 & TND & $\mathrm{RN}$ & 5 & 39.75 & 3.9853 \\
\hline 98 & 44 & Male & $|-| \mid$ & T1NOMO & Clear cell & None & 227 & $<40$ & PN & 1 & 50.3 & 1.0147 \\
\hline 99 & 61 & Male & $|-| \mid$ & T3NOMO & Clear cell & None & 621 & 44090 & $\mathrm{RN}$ & 2 & 45.95 & 2.6584 \\
\hline 100 & 56 & Female & $|-| \mid$ & T1NOMO & Clear cell & None & 591 & TND & PN & 4 & 38.9 & 4.3251 \\
\hline 101 & 52 & Male & III-IV & T1N0M0 & Clear cell & None & 790 & NT & $\mathrm{RN}$ & 2 & 45.45 & 2.7892 \\
\hline 102 & 44 & Male & $|-| \mid$ & T1NOMO & Clear cell & None & 544 & TND & PN & 3 & 40.3 & 3.4663 \\
\hline 103 & 51 & Male & III-IV & T1NOMO & Clear cell & Diabetes & 980 & $<40$ & $\mathrm{RN}$ & 2 & 44.3 & 2.6835 \\
\hline 104 & 38 & Male & III-IV & T1NOMO & Clear cell & None & 555 & 399 & $\mathrm{RN}$ & 5 & 41.15 & 3.4678 \\
\hline 105 & 57 & Male & III-IV & T3NOMO & Clear cell & None & 288 & TND & $\mathrm{RN}$ & 2 & 49.15 & 0.9785 \\
\hline 106 & 37 & Female & $|-| \mid$ & T1N0M0 & Clear cell & Hypertension & 543 & TND & PN & 1 & 48 & 2.4318 \\
\hline
\end{tabular}

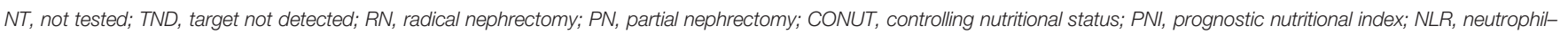
lymphocyte ratio.

information than RNA levels and CD4 counts. Low levels of serum albumin not only reflect the general health status of HIV-infected patients but also reflect the effects of HIV on the host (30). Several studies of HIV seroepidemic cohorts have shown that low serum albumin is associated with all-cause mortality, even among individuals receiving $\operatorname{HAART}(31,32)$. Sabin et al. demonstrated an independent role of serum albumin detected shortly after HIV serotransformation in all-cause mortality and a smaller but still significant role in AIDS progression. These associations were independent of CD4+ T lymphocyte count and HIV viral load (33).
Lymphocytes are believed to have antitumor ability by affecting the growth, migration, and apoptosis and inducing the cytotoxicity of tumor cells. The high density of lymphocytes reflects the immune response of tumors (34). F A Post et al. found that total lymphocyte count and CD4+ T lymphocyte count were equally good predictors of HIV infection disease progression, and severe lymphocytopenia (total lymphocyte counts $<750 / \mu \mathrm{l}$ ) predicted low survival and may reflect high susceptibility to opportunistic infections $(35,36)$. Moses $\mathrm{R}$ Kamya's results showed a strong correlation between total lymphocyte counts 
TABLE 3 | Clinicopathological characteristics of the 106 patients according to different CONUT groups.

\begin{tabular}{|c|c|c|c|c|}
\hline Factors & $\begin{array}{c}\text { Total } \\
(n=106)\end{array}$ & $\begin{array}{c}\text { CONUT<3 } \\
(n=61)\end{array}$ & $\begin{array}{c}\text { CONUT } \geq 3 \\
(n=45)\end{array}$ & $P$-value* \\
\hline \multicolumn{5}{|l|}{ Age (years) } \\
\hline$\leq 65$ & 94 & 55 & 39 & \multirow[t]{2}{*}{$P=0.574$} \\
\hline$>65$ & 12 & 6 & 6 & \\
\hline \multicolumn{5}{|l|}{ Gender } \\
\hline Male & 93 & 54 & 39 & \multirow[t]{2}{*}{$P=0.773$} \\
\hline Female & 13 & 7 & 6 & \\
\hline \multicolumn{5}{|c|}{ Hypertention } \\
\hline Yes & 14 & 10 & 4 & \multirow[t]{2}{*}{$P=0.259$} \\
\hline No & 92 & 51 & 41 & \\
\hline \multicolumn{5}{|l|}{ Diabetes } \\
\hline Yes & 11 & 8 & 3 & \multirow[t]{2}{*}{$P=0.451$} \\
\hline No & 95 & 53 & 42 & \\
\hline \multicolumn{5}{|c|}{ Tumor grade } \\
\hline T1NOMO & 87 & 50 & 37 & \multirow[t]{2}{*}{$P=0.973$} \\
\hline T3NOMO & 19 & 11 & 8 & \\
\hline \multicolumn{5}{|c|}{ Fuhrman grade } \\
\hline$|-| \mid$ & 65 & 43 & 22 & \multirow[t]{2}{*}{$P=0.024$} \\
\hline III-IV & 41 & 18 & 23 & \\
\hline \multicolumn{5}{|l|}{ Histology } \\
\hline Clear cell & 104 & 60 & 44 & \multirow[t]{2}{*}{$P=0.671$} \\
\hline Non-clear & 2 & 1 & 1 & \\
\hline \multicolumn{5}{|l|}{ cell } \\
\hline \multicolumn{5}{|l|}{ Surgery } \\
\hline RN & 80 & 46 & 34 & \multirow[t]{2}{*}{$P=0.986$} \\
\hline PN & 26 & 15 & 11 & \\
\hline \multicolumn{5}{|c|}{ CD4+ T lymphocyte count (cells/ul) } \\
\hline$\geq 200$ & 95 & 56 & 39 & \multirow[t]{2}{*}{$P=0.593$} \\
\hline$<200$ & 11 & 5 & 6 & \\
\hline
\end{tabular}

${ }^{*}$ Chi-square test. Bold value indicates statistical significance in univariate and multivariate analysis which had been detailed in the "Results" section.

$R N$, radical nephrectomy; PN, partial nephrectomy.

and CD4+ T lymphocytes. Similar correlations between total lymphocyte counts and CD4+ T lymphocytes have been reported in North America, England and India (37).
Low cholesterol levels are associated with cancer outcomes. Cholesterol affects the structure and function of the membrane, such as membrane protein activity and membrane fluidity, thus affecting the ability of immunoactive cells to fight cancer cells (38). Dyslipidemia has also been observed in untreated HIVinfected patients, suggesting that HIV infection itself has deleterious metabolic effects (39). In the Swiss HIV cohort study, the use of HIV protease inhibitors was found to be associated with an increase in plasma total cholesterol (40). In the SMART study, discontinuation of HAART led to a reduction in total cholesterol concentration (41). After the initiation of HAART, lipid abnormalities in HIV patients become more obvious, and hypercholesterolemia is the most related disease (42-44). Serum total cholesterol concentration was a correlated and independent predictor of HIV RNA load, CD4+ T lymphocyte count and WHO clinical stage. In this era of testing and treatment, it is possible to use low serum total cholesterol concentration as a marker to predict the efficacy of HAART (45).

CONUT is mainly associated with malignant tumors and survival prognosis through the nutritional immune pathway. For $\mathrm{HIV}$-infected patients, nutritional and immune functions are already low, and these patients have an increased risk of RCC, which is largely due to the loss of control of the oncogenic genome and the high prevalence of exposure to other carcinogens (46). Adam B Murphy et al. found that the frequency of metastatic disease in an HIV-related cohort was 2.5 times that observed in a non-HIV-related cohort, although this difference did not reach statistical significance (47). Wee Loon ONG's study showed that a total of five HIV-related RCC patients in Australia's statewide HIV centers underwent surgery without any perioperative complications (48). In metastatic clear cell RCC, targeted therapy or immunotherapy may interact with antiretroviral drugs to some extent (49). Annah B Layman et al. found a similar incidence, clinical presentation and outcome of
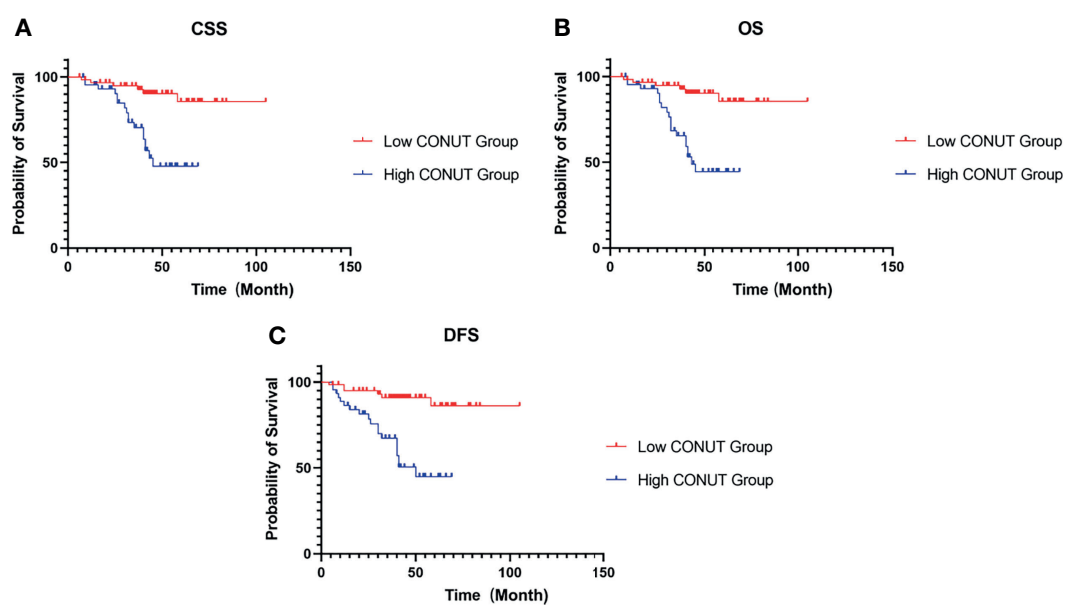

FIGURE 2 | Kaplan-Meier survival curves for HIV-related RCC patients treated with surgery. Survival curves set at cutoff value 3 for CSS (A), OS (B) and DFS (C) RCC, renal cell carcinoma; CSS, cancer-specific survival; OS, overall survival; DFS, disease-free survival; CONUT, controlling nutritional status. 
TABLE 4 | Univariate and multivariate analyses of clinicopathological parameters to predict CSS, OS and DFS in patients with HIV-related RCC.

\begin{tabular}{|c|c|c|c|c|c|c|c|c|c|c|c|c|}
\hline \multirow[t]{3}{*}{ Factors } & \multicolumn{4}{|c|}{ css } & \multicolumn{4}{|c|}{ os } & \multicolumn{4}{|c|}{ DFS } \\
\hline & \multicolumn{2}{|c|}{ Univariate } & \multicolumn{2}{|c|}{ Multivariate } & \multicolumn{2}{|c|}{ Univariate } & \multicolumn{2}{|c|}{ Multivariate } & \multicolumn{2}{|c|}{ Univariate } & \multicolumn{2}{|c|}{ Multivariate } \\
\hline & $\begin{array}{c}\text { HR } \\
(95 \% \mathrm{Cl})\end{array}$ & $\begin{array}{c}P \text { - } \\
\text { value }\end{array}$ & $\begin{array}{c}\text { HR } \\
(95 \% \mathrm{Cl})\end{array}$ & $\begin{array}{c}P \text { - } \\
\text { value }\end{array}$ & $\begin{array}{c}\text { HR } \\
(95 \% \mathrm{Cl})\end{array}$ & $\begin{array}{c}P- \\
\text { value }\end{array}$ & $\begin{array}{c}\text { HR } \\
(95 \% \mathrm{Cl})\end{array}$ & $\begin{array}{c}P \text { - } \\
\text { value }\end{array}$ & $\begin{array}{c}\text { HR } \\
(95 \% \mathrm{Cl})\end{array}$ & $\begin{array}{c}P \text { - } \\
\text { value }\end{array}$ & $\begin{array}{c}\text { HR } \\
(95 \% \mathrm{Cl})\end{array}$ & $\begin{array}{c}P \text { - } \\
\text { value }\end{array}$ \\
\hline \multicolumn{13}{|l|}{ Age } \\
\hline$>65$ & $\begin{array}{c}1.612(0.548- \\
4.745)\end{array}$ & 0.386 & & & $\begin{array}{c}1.473(0.505- \\
4.96)\end{array}$ & 0.479 & & & $\begin{array}{c}1.517(0.520- \\
4.423)\end{array}$ & 0.446 & & \\
\hline$\leq 65$ & 1.00 (ref) & & & & 1.00 (ref) & & & & 1.00 (ref) & & & \\
\hline \multicolumn{13}{|l|}{ Gender } \\
\hline Male & $\begin{array}{c}23.809(0.057- \\
9932.7)\end{array}$ & 0.303 & & & $\begin{array}{c}23.826(0.075- \\
7603.4)\end{array}$ & 0.281 & & & $\begin{array}{c}2.959(0.399- \\
21.933)\end{array}$ & 0.289 & & \\
\hline Female & 1.00 (ref) & & & & 1.00 (ref) & & & & 1.00 (ref) & & & \\
\hline \multicolumn{13}{|l|}{ Fuhrman grade } \\
\hline$|-| \mid$ & $\begin{array}{c}0.086(0.028- \\
0.257)\end{array}$ & $<0.01$ & $\begin{array}{c}0.192 \\
(0.045- \\
0.810)\end{array}$ & 0.025 & $\begin{array}{c}0.079(0.026- \\
0.234)\end{array}$ & $<0.01$ & $\begin{array}{c}0.203 \\
(0.049- \\
0.842)\end{array}$ & 0.028 & $\begin{array}{c}0.104(0.038- \\
0.282)\end{array}$ & $<0.01$ & $\begin{array}{c}0.180 \\
(0.048- \\
0.669)\end{array}$ & 0.010 \\
\hline III-IV & 1.00 (ref) & & 1.00 (ref) & & 1.00 (ref) & & 1.00 (ref) & & 1.00 (ref) & & 1.00 (ref) & \\
\hline \multicolumn{13}{|l|}{ Tumor grade } \\
\hline T1NOMO & $\begin{array}{l}0.757(0.281- \\
2.042)\end{array}$ & 0.583 & & & $\begin{array}{c}0.673(0.268- \\
1.687)\end{array}$ & 0.398 & & & $\begin{array}{c}0.823(0.308- \\
2.195)\end{array}$ & 0.697 & & \\
\hline T3NOMO & 1.00 (ref) & & & & 1.00 (ref) & & & & 1.00 (ref) & & & \\
\hline \multicolumn{13}{|l|}{ Histology } \\
\hline Clear cell & $\begin{array}{c}20.919(0.000- \\
2325088)\end{array}$ & 0.608 & & & $\begin{array}{c}20.917(0.000- \\
1434880)\end{array}$ & 0.593 & & & $\begin{array}{c}20.908(0.000- \\
1589742)\end{array}$ & 0.596 & & \\
\hline Non-clear cell & 1.00 (ref) & & & & 1.00 (ref) & & & & 1.00 (ref) & & & \\
\hline \multicolumn{13}{|l|}{ Hypertension } \\
\hline Yes & $\begin{array}{c}1.213(0.360- \\
4.087)\end{array}$ & 0.755 & & & $\begin{array}{c}1.109(0.332- \\
3.709)\end{array}$ & 0.867 & & & $\begin{array}{c}1.044(0.312- \\
3.491)\end{array}$ & 0.945 & & \\
\hline No & 1.00 (ref) & & & & 1.00 (ref) & & & & 1.00 (ref) & & & \\
\hline \multicolumn{13}{|l|}{ Diabetes } \\
\hline Yes & $\begin{array}{c}0.862(0.202- \\
3.680)\end{array}$ & 0.842 & & & $\begin{array}{c}0.790(0.186- \\
3.352)\end{array}$ & 0.749 & & & $\begin{array}{c}0.782(0.184- \\
3.317)\end{array}$ & 0.738 & & \\
\hline No & 1.00 (ref) & & & & 1.00 (ref) & & & & 1.00 (ref) & & & \\
\hline \multicolumn{13}{|l|}{ Surgery } \\
\hline $\mathrm{RN}$ & $\begin{array}{c}2.247(0.763- \\
6.615)\end{array}$ & 0.142 & & & $\begin{array}{c}2.478(0.849- \\
7.229)\end{array}$ & 0.097 & & & $\begin{array}{c}1.760(0.660- \\
4.694)\end{array}$ & 0.259 & & \\
\hline PN & 1.00 (ref) & & & & 1.00 (ref) & & & & 1.00 (ref) & & & \\
\hline \multicolumn{13}{|c|}{ CD4 count (cells/ul) } \\
\hline$\geq 200$ & $\begin{array}{c}0.663(0.225- \\
1.951)\end{array}$ & 0.455 & & & $\begin{array}{c}0.736(0.252- \\
2.147)\end{array}$ & 0.575 & & & $\begin{array}{c}0.720(0.247- \\
2.099)\end{array}$ & 0.547 & & \\
\hline$<200$ & 1.00 (ref) & & & & 1.00 (ref) & & & & 1.00 (ref) & & & \\
\hline \multicolumn{13}{|l|}{ CONUT score } \\
\hline$<3$ & $\begin{array}{c}0.197(0.077- \\
0.502)\end{array}$ & 0.001 & $\begin{array}{c}0.225 \\
(0.067- \\
0.749)\end{array}$ & 0.015 & $\begin{array}{c}0.177(0.070- \\
0.446)\end{array}$ & $<0.001$ & $\begin{array}{c}0.201 \\
(0.061- \\
0.661)\end{array}$ & 0.008 & $\begin{array}{c}0.176(0.070- \\
0.444)\end{array}$ & $<0.001$ & $\begin{array}{c}0.227 \\
(0.078- \\
0.664)\end{array}$ & 0.007 \\
\hline$\geq 3$ & 1.00 (ref) & & 1.00 (ref) & & 1.00 (ref) & & 1.00 (ref) & & 1.00 (ref) & & 1.00 (ref) & \\
\hline
\end{tabular}

Bold values indicate statistical significance in univariate and multivariate analysis which had been detailed in the "Results" section.

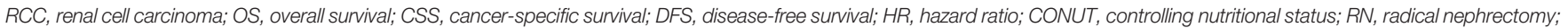

PN, partial nephrectomy.

RCC in HIV-infected and non-HIV-infected populations and no association between CD4+ T lymphocyte count and RCC risk at the onset of AIDS (50).

Our study has some limitations. First, the follow-up time of some patients was short, at only half a year, so it would be more meaningful to extend the follow-up time to ensure the accuracy of the results. Furthermore, the duration of treatment with antiviral drugs may also be a prognostic factor, but since most of the included patients were uncertain about the duration of HAART, no reliable data were obtained.

\section{CONCLUSION}

HIV-infected patients eligible for HAART have a potentially normal life expectancy. Therefore, diseases such as RCC and other malignancies should be treated in the same way as those in non-HIV-infected patients. As it becomes increasingly possible to operate on HIV-infected patients undergoing HAART, CONUT's role in predicting survival for HIV-related RCC is becoming increasingly important. The preoperative CONUT score not only objectively reflects the nutritional and immune 
statuses of the host but also is an independent predictor of CSS, OS and DFS in patients with HIV-related RCC.

\section{DATA AVAILABILITY STATEMENT}

The raw data supporting the conclusions of this article will be made available by the authors, without undue reservation.

\section{ETHICS STATEMENT}

The studies involving human participants were reviewed and approved by Beijing You'an Hospital Affiliated to Capital

\section{REFERENCES}

1. Capitanio U, Montorsi F. Renal Cancer. Lancet (2016) 387(10021):894-906. doi: 10.1016/S0140-6736(15)00046-X

2. Ljungberg B, Bensalah K, Canfield S, Dabestani S, Hofmann F, Hora M, et al. EAU Guidelines on Renal Cell Carcinoma: 2014 Update. Eur Urol (2015) 67 (5):913-24. doi: 10.1016/j.eururo.2015.01.005

3. Martín-Moreno A, Muñoz-Fernández MA. Dendritic Cells, the Double Agent in the War Against HIV-1. Front Immunol (2019) 10:2485. doi: 10.3389/ fimmu.2019.02485

4. Heyns CF, Smit SG, van der Merwe A, Zarrabi AD. Urological Aspects of HIV and AIDS. Nat Rev Urol (2013) 10(12):713-22. doi: 10.1038/nrurol.2013.230

5. Chen CH, Chung CY, Wang LH, Lin C, Lin HL, Lin HC. Risk of Cancer Among HIV-Infected Patients From a Population-Based Nested CaseControl Study: Implications for Cancer Prevention. BMC Cancer (2015) 15:133. doi: 10.1186/s12885-015-1099-y

6. Melku M, Abebe G, Teketel A, Asrie A, Yalew A, Biadgo A, et al. Immunological Status and Virological Suppression Among HIV-Infected Adults on Highly Active Antiretroviral Therapy. Environ Health Prev Med (2020) 25(1):43. doi: 10.1186/s12199-020-00881-6

7. Crum-Cianflone N, Hullsiek KH, Marconi V, Weintrob A, Ganesan A, Barthel RV, et al. Trends in the Incidence of Cancers Among HIV-Infected Persons and the Impact of Antiretroviral Therapy: A 20-Year Cohort Study. AIDS (2009) 23(1):41-50. doi: 10.1097/QAD.0b013e328317cc2d

8. Zheng Y, Bao L, Wang W, Wang Q, Pan Y, Gao X. Prognostic Impact of the Controlling Nutritional Status Score Following Curative Nephrectomy for Patients With Renal Cell Carcinoma. Medicine (Baltimore) (2018) 97(49): e13409. doi: 10.1097/MD.0000000000013409. [Published Correction Appears in Medicine (Baltimore). 2019 Jan;98(2):e14200].

9. Wang J, Liu Y, Mi X, Shao M, Liu L. The Prognostic Value of Prognostic Nutritional Index (PNI) and Neutrophil to Lymphocyte Ratio (NLR) for Advanced non-Small Cell Lung Cancer Treated With Platinum-Based Chemotherapeutics. Ann Palliat Med (2020) 9(3):967-78. doi: 10.21037/ apm.2020.04.31

10. Miyamoto R, Inagawa $S$, Sano N, Tadano S, Adachi S, Yamamoto M. The Neutrophil- to-Lymphocyte Ratio (NLR) Predicts Short-Term and LongTerm Outcomes in Gastric Cancer Patients. Eur J Surg Oncol (2018) 44 (5):607-12. doi: 10.1016/j.ejso.2018.02.003

11. Hua X, Long ZQ, Huang X, Deng JP, He ZY, Guo L, et al. The Value of Prognostic Nutritional Index (PNI) in Predicting Survival and Guiding Radiotherapy of Patients With T1-2n1 Breast Cancer. Front Oncol (2020) 9:1562. doi: $10.3389 /$ fonc. 2019.01562

12. Mirili C, Yılmaz A, Demirkan S, Bilici M, Basol Tekin S. Clinical Significance of Prognostic Nutritional Index (PNI) in Malignant Melanoma. Int J Clin Oncol (2019) 24(10):1301-10. doi: 10.1007/s10147-019-01461-7

13. Toyokawa T, Kubo N, Tamura T, Sakurai K, Amano R, Tanaka H, et al. The Pretreatment Controlling Nutritional Status (CONUT) Score Is an Independent Prognostic Factor in Patients With Resectable Thoracic Esophageal Squamous Cell Carcinoma: Results From a Retrospective Study. BMC Cancer (2016) 16(1):722. doi: 10.1186/s12885-016-2696-0
Medical University. The patients/participants provided their written informed consent to participate in this study.

\section{AUTHOR CONTRIBUTIONS}

WX participated in manuscript preparation and writing. $\mathrm{XH}$ provided suggestion and edits. YZ (4th author) conceptualized, wrote, and revised manuscript. YZ (2nd author) and HW provided relevant patients data of their hospitals and offered suggestions for revising the article. All authors contributed to the article and approved the submitted version.

14. Somarriba G, Neri D, Schaefer N, Miller TL. The Effect of Aging, Nutrition, and Exercise During HIV Infection. HIV AIDS (Auckl) (2010) 2:191-201. doi: $10.2147 /$ HIV.S9069

15. De Carvalho BF, Policarpo S, Moreira AC. Nutritional Status and Quality of Life in HIV-Infected Patients. Nutr Hosp (2017) 34(4):923-33. doi: 10.20960/nh.453

16. Apornpong T, Han WM, Chattranukulchai P, Siwamogsatham S, Wattanachanya L, Gatechompol S, et al. Higher Proportion of Abnormal Nutritional Status Among Well-Suppressed HIV-Infected Elderly Asians Compared to HIV-Negative Individuals. AIDS Res Hum Retroviruses (2020) 36(7):590-6. doi: 10.1089/AID.2019.0285

17. Colecraft E. HIV/AIDS: Nutritional Implications and Impact on Human Development. Proc Nutr Soc (2008) 67(1):109-13. doi: 10.1017/ S0029665108006095

18. Prado CM, Wells JC, Smith SR, Stephan BC, Siervo M. Sarcopenic Obesity: A Critical Appraisal of the Current Evidence. Clin Nutr (2012) 31(5):583-601. doi: 10.1016/j.clnu.2012.06.010

19. Ishihara H, Kondo T, Yoshida K, Omae K, Takagi T, Lizuka J, et al. Preoperative Controlling Nutritional Status (CONUT) Score as a Novel Predictive Biomarker of Survival in Patients With Localized Urothelial Carcinoma of the Upper Urinary Tract Treated With Radical Nephroureterectomy. Urol Oncol (2017) 35 (9):539.e9-16. doi: 10.1016/j.urolonc.2017.04.012

20. Kang HW, Seo SP, Kim WT, Yun SJ, Lee SC, Kim WJ, et al. Prognostic Impact of Nutritional Status Assessed by the Controlling Nutritional Status (CONUT) Score in Patients With Surgically Treated Renal Cell Carcinoma. Nutr Cancer (2018) 70(6):886-94. doi: 10.1080/01635581.2018.1490448

21. Xu H, Tan P, Jin X, Ai J, Lin T, Lei H, et al. Validation of the Preoperative Controlling Nutritional Status Score as an Independent Predictor in a Large Chinese Cohort of Patients With Upper Tract Urothelial Carcinoma. Cancer Med (2018) 7(12):6112-23. doi: 10.1002/cam4.1902

22. Elghiaty A, Kim J, Jang WS, Park JS, Heo JE, Rha KH, et al. Preoperative Controlling Nutritional Status (CONUT) Score as a Novel Immune-Nutritional Predictor of Survival in non-Metastatic Clear Cell Renal Cell Carcinoma of $\leq 7 \mathrm{Cm}$ on Preoperative Imaging. J Cancer Res Clin Oncol (2019) 145(4):957-65. doi: 10.1007/s00432-019-02846-x

23. Song H, Xu B, Luo C, Zhang Z, Ma B, Jin J, et al. The Prognostic Value of Preoperative Controlling Nutritional Status Score in Non-Metastatic Renal Cell Carcinoma Treated With Surgery: A Retrospective Single-Institution Study. Cancer Manag Res (2019) 11:7567-75. doi: 10.2147/CMAR.S209418

24. Bao Z, Li Y, Guan B, Xiong G, Zhang L, Tang Q, et al. High Preoperative Controlling Nutritional Status Score Predicts a Poor Prognosis in Patients With Localized Upper Tract Urothelial Cancer: A Propensity Score Matching Study in a Large Chinese Center. Cancer Manag Res (2020) 12:323-35. doi: 10.2147/CMAR.S225711

25. Takemura K, Yuasa T, Fujiwara R, Ito M, Suzuki H, Yonese J, et al. Prognostic Significance of the Controlling Nutritional Status (CONUT) Score in Patients With Advanced Renal Cell Carcinoma Treated With Nivolumab After Failure of Prior Tyrosine Kinase Inhibitors. J Urol (2020) 204(6):1166-72. doi: 10.1097/JU.0000000000001196

26. Smith CJ, Ryom L, Weber R, Morlat P, Pradier C, Reiss P, et al. Trends in Underlying Causes of Death in People With HIV From 1999 to 2011 (D:A:D): 
A Multicohort Collaboration. Lancet (2014) 384(9939):241-8. doi: 10.1016/ S0140-6736(14)60604-8

27. Harris HW, Schecter WP. Surgical Risk Assessment and Management in Patients With HIV Disease. Gastroenterol Clin N Am (1997) 26(2):377-91. doi: 10.1016/s0889-8553(05)70300-9

28. Robich MP, Schiltz N, Johnston DR, Mick S, Tse W, Koch C, et al. Outcomes of Patients With Human Immunodeficiency Virus Infection Undergoing Cardiovascular Surgery in the United States. J Thorac Cardiovasc Surg (2014) 148(6):3066-73. doi: 10.1016/j.jtcvs.2014.07.074

29. Tanriverdi O. A Discussion of Serum Albumin Level in Advanced-Stage Hepatocellular Carcinoma: A Medical Oncologist's Perspective. Med Oncol (2014) 31(11):282. doi: 10.1007/s12032-014-0282-3

30. Mehta SH, Astemborski J, Sterling TR, Thomas DL, Vlahov D. Serum Albumin as a Prognostic Indicator for HIV Disease Progression. AIDS Res Hum Retroviruses (2006) 22(1):14-21. doi: 10.1089/aid.2006.22.14

31. Feldman JG, Gange SJ, Bacchetti P, Cohen M, Young M, Squires KE, et al. Serum Albumin Is a Powerful Predictor of Survival Among HIV-1-Infected Women. J Acquir Immune Defic Syndr (2003) 33(1):66-73. doi: 10.1097/ 00126334-200305010-00010

32. Sudfeld CR, Isanaka S, Aboud S, Mugusi FM, Wang M, Chalamilla GE, et al. Association of Serum Albumin Concentration With Mortality, Morbidity, CD4 T-Cell Reconstitution Among Tanzanians Initiating Antiretroviral Therapy. J Infect Dis (2013) 207(9):1370-8. doi: 10.1093/infdis/jit027

33. Sabin CA, Griffioen A, Yee TT, Emery VC, Herrero-Martinez E, Phillips AN, et al. Markers of HIV-1 Disease Progression in Individuals With Haemophilia Coinfected With Hepatitis C Virus: A Longitudinal Study. Lancet (2002) 360 (9345):1546-51. doi: 10.1016/S0140-6736(02)11519-4

34. Mantovani A, Allavena P, Sica A, Balkwill F. Cancer-Related Inflammation. Nature (2008) 454(7203):436-44. doi: 10.1038/nature07205

35. Post FA, Wood R, Maartens G. CD4 and Total Lymphocyte Counts as Predictors of HIV Disease Progression. QJM (1996) 89(7):505-8. doi: 10.1093/qjmed/89.7.505

36. Lau B, Gange SJ, Phair JP, Riddler SA, Detels R, Margolick JB. Rapid Declines in Total Lymphocyte Counts and Hemoglobin Concentration Prior to AIDS Among HIV-1-Infected Men. AIDS (2003) 17(14):2035-44. doi: 10.1097/ 00002030-200309260-00004

37. Kamya MR, Semitala FC, Quinn TC, Ronald A, Njama-Meya D, MayanjaKizza H, et al. Total Lymphocyte Count of 1200 Is Not a Sensitive Predictor of CD4 Lymphocyte Count Among Patients With HIV Disease in Kampala, Uganda. Afr Health Sci (2004) 4(2):94-101.

38. Resnik N, Sepcic K, Plemenitas A, Windoffer R, Leube R, Veranic P. Desmosome Assembly and Cell-Cell Adhesion are Membrane Raft-Dependent Processes. J Biol Chem (2011) 286(2):1499-507. doi: 10.1074/jbc.M110.189464

39. Fisher SD, Miller TL, Lipshultz SE. Impact of HIV and Highly Active Antiretroviral Therapy on Leukocyte Adhesion Molecules, Arterial Inflammation, Dyslipidemia, and Atherosclerosis. Atherosclerosis (2006) 185 (1):1-11. doi: 10.1016/j.atherosclerosis.2005.09.025

40. Périard D, Telenti A, Sudre P, Cheseaux JJ, Halfon P, Reymond MJ, et al. Atherogenic Dyslipidemia in HIV-Infected Individuals Treated With Protease Inhibitors. The Swiss HIV Cohort Study. Circulation (1999) 100(7):700-5. doi: 10.1161/01.cir.100.7.700

41. Strategies for Management of Antiretroviral Therapy (SMART) Study Group, El-Sadr WM, Lundgren J, Neaton JD, Gordin F, Abrams D, et al. CD4+ Count-Guided Interruption of Antiretroviral Treatment. N Engl J Med (2006) 355(22):2283-96. doi: 10.1056/NEJMoa062360
42. Sposito AC, Caramelli B, Fonseca FA, Bertolami MC, Neto AA, Souza AD, et al. IV Diretriz Brasileira Sobre Dislipidemias E Prevenção Da Aterosclerose: Departamento De Aterosclerose Da Sociedade Brasileira De Cardiologia [IV Brazilian Guideline for Dyslipidemia and Atherosclerosis Prevention: Department of Atherosclerosis of Brazilian Society of Cardiology]. Arq Bras Cardiol (2007) 88 Suppl 1:2-19. doi: 10.1590/s0066-782x2007000700002

43. Mulligan K, Grunfeld C, Tai VW, Algren H, Pang M, Chernoff DN, et al. Hyperlipidemia and Insulin Resistance are Induced by Protease Inhibitors Independent of Changes in Body Composition in Patients With HIV Infection. J Acquir Immune Defic Syndr (2000) 23(1):35-43. doi: 10.1097/ 00126334-200001010-00005

44. Savès M, Raffi F, Capeau J, Rozenbaum W, Ragnaud JM, Perronne C, et al. Factors Related to Lipodystrophy and Metabolic Alterations in Patients With Human Immunodeficiency Virus Infection Receiving Highly Active Antiretroviral Therapy. Clin Infect Dis (2002) 34(10):1396-405. doi: $10.1086 / 339866$

45. Adal M, Howe R, Kassa D, Aseffa A, Petros B. Associations of Gender and Serum Total Cholesterol With CD4+ T Cell Count and HIV RNA Load in Antiretroviral-Naïve Individuals in Addis Ababa. BMC Public Health (2018) 18(1):943. doi: 10.1186/s12889-018-5852-4

46. Intra M, Gentilini O, Brenelli F, Chagas EM, Veronesi U, Sandri MT. Breast Cancer Among HIV-Infected Patients: The Experience of the European Institute of Oncology. J Surg Oncol (2005) 91(2):141-2. doi: 10.1002/jso.20315

47. Murphy AB, Bhatia R, Martin IK, Klein DA, Hollowell CM, Nyame Y, et al. Are HIV-Infected Men Vulnerable to Prostate Cancer Treatment Disparities? Cancer Epidemiol Biomarkers Prev (2014) 23(10):2009-18. doi: 10.1158/10559965.EPI-14-0614

48. Ong WL, King K, Koh TL, Chipman M, Royce P, Hoy J, et al. HIV and Renal Cell Carcinoma: Experience in an Australian Statewide HIV Center. Asia Pac J Clin Oncol (2016) 12(2):188-93. doi: 10.1111/ajco.12487

49. Deeken JF, Pantanowitz L, Dezube BJ. Targeted Therapies to Treat nonAIDS-Defining Cancers in Patients With HIV on HAART Therapy: Treatment Considerations and Research Outlook. Curr Opin Oncol (2009) 21(5):445-54. doi: 10.1097/CCO.0b013e32832f3e04

50. Layman AB, Engels EA. Kidney and Bladder Cancers Among People With AIDS in the United States. J Acquir Immune Defic Syndr (2008) 48(3):365-7. doi: 10.1097/QAI.0b013e31817ae5da

Conflict of Interest: The authors declare that the research was conducted in the absence of any commercial or financial relationships that could be construed as a potential conflict of interest.

Publisher's Note: All claims expressed in this article are solely those of the authors and do not necessarily represent those of their affiliated organizations, or those of the publisher, the editors and the reviewers. Any product that may be evaluated in this article, or claim that may be made by its manufacturer, is not guaranteed or endorsed by the publisher.

Copyright $\odot 2021$ Xue, Zhang, Wang, Zhang and Hu. This is an open-access article distributed under the terms of the Creative Commons Attribution License (CC BY). The use, distribution or reproduction in other forums is permitted, provided the original author(s) and the copyright owner(s) are credited and that the original publication in this journal is cited, in accordance with accepted academic practice. No use, distribution or reproduction is permitted which does not comply with these terms. 